\title{
ZNS-Befall bei DLBCL-Patienten
}

Ein Ann-Arbor-Stadium von 3 oder 4, Albumin-Spiegel unter 3,2 mg/l, ein Befall retroperitonealer Lymphknoten sowie mehr als ein extranodaler Befund eignen sich bei Patienten mit diffusem großzelligem B-Zell-Lymphom (DLBCL) als Parameter für die Risikostratifizierung eines ZNS-Rückfalls, wie eine japanische Studie ergab. Dabei handelt es sich um eine retrospektive Auswertung der Daten von 413 Patienten mit einer neu diagnostizierten Erkrankung, die sich einer R-CHOP oder vergleichbaren Behandlung am Komagome Krankenhaus in Tokio unterzogen hatten.

Ein ZNS-Rückfall trat bei $6,5 \%$ der Patienten auf. Die 5-Jahres-Wahrscheinlichkeit für einen ZNS-Rückfall betrug $8,4 \%$, die mediane Zeit von der Diagnosestellung bis zum ZNS-Progress $15 \mathrm{Mo-}$ nate und das mediane Überleben nach Auftreten der ZNS-Metastasen sieben Monate. In univariaten Analysen ergaben sich als Risikofaktoren für ZNS-Metasta- sen ein Ann-Arbor-Stadium von 3 oder 4, ein Albumin-Spiegel unter 3,2 mg/l, mehr als ein extranodaler Befund sowie die Beteiligung von retroperitonealen Lymphknoten.

Diese vier Risikofaktoren dienten als Grundlage für ein prognostisches Modell. Demnach ist die 5-Jahres-Wahrscheinlichkeit für einen ZNS-Rückfall signifikant höher bei Patienten, bei denen mindestens drei dieser vier Risikofaktoren vorliegen, im Vergleich zu Patienten mit zwei oder weniger dieser Risikofaktoren $(26,4$ vs. $3,0 \%$; p < 0,001). Das Risikomodell eignete sich gut zur Risikostratifizierung und könnte helfen, Hochrisikopatienten zu identifizieren, für die eine ZNS-Prophylaxe gerechtfertigt ist.

Barbara Kreutzkamp

Kanemasa Y et al. Central nervous system relapse in patients with diffuse large B cell lymphoma: analysis of the risk factors and proposal of a new prognostic model. Ann Hematol. 2016;95(10):1661-9.

\section{kurz notiert}

Forschungsstandort Deutschland

p53-Mutationen und Metastasierung +++ p53-Mutationen können das Gen der Ektonukleosidtriphosphat-Diphosphohydrolase 5 (ENTPD5) im endoplasmatischen Retikulum aktivieren und so die Faltung von $\mathrm{N}$-glykosylierten Membranproteinen fördern. ENTPD5 fungiert dabei als Uridin-5-Diphosphatase (UDPase). Das fanden Forscher der Universität Marburg heraus. Wurde ENTPD5 im Tiermodell ausgeschaltet, so bildeten sich keine Metastasen in der Lunge. Dies belegt, dass ENTPD5 für die Metastasenbildung erforderlich ist [Vogiatzia F et al. Proc Natl Acad Sci. 2016;113(52):E8433-42].

red.

Neue Ziele für die Immuntherapie +++ Bei der Immuntherapie von Tumoren kommt es darauf an, herauszufinden, welche Moleküle das Immunsystem auf Krebszellen erkennt. Einem Forscherteam aus München ist es nun erstmals gelungen, geeignete Proteinstrukturen (mutierte Peptidliganden) im Tumorgewebe von Hautkrebspatienten direkt zu identifizieren. Dabei verwendeten sie die Massenspektrometrie. Diese Methode könnte künftig genutzt werden, um Neoepitope mit hoher Relevanz für immuntherapeutische Strategien zu finden [Bassani-Sternberg M et al. Nat Commun. 2016; 7:13404]

red.

\section{Schilddrüsenkarzinom}

\section{Wie viele Lymphknoten untersuchen?}

Das Risiko für einen postoperativ nicht erkannten Lymphknotenbefall bei Patienten mit papillärem Schilddrüsenkarzinom ist vom Stadium des primären Tumors und der Anzahl untersuchter Lymphknoten abhängig. Das fanden US-amerikanische Forscher heraus. Bei Patienten mit papillärem Schilddrüsenkarzinom besteht nach der Operation ein

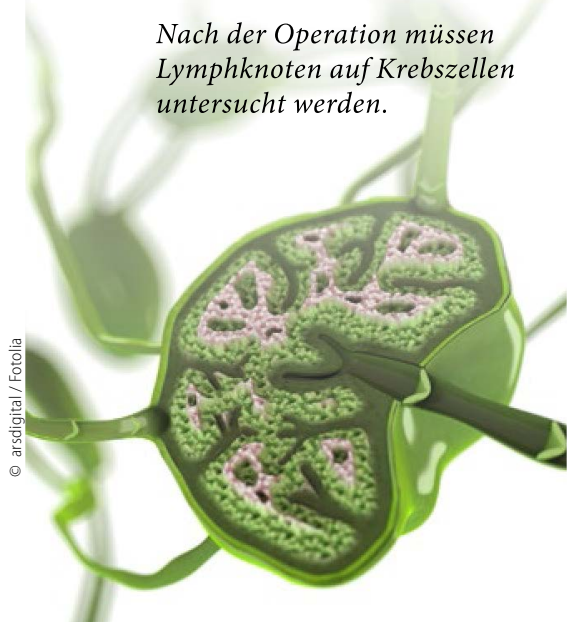

Risiko, dass Lymphknotenmetastasen übersehen wurden. Dies könnte besonders gelten, wenn die Lymphknoten nur eingeschränkt untersucht wurden. Deshalb analysierten die Wissenschaftler, wie hoch das Risiko für übersehene positive Lymphknoten bei Patienten mit lokalisiertem, $\geq 1 \mathrm{~cm}$ großem papillärem Schilddrüsenkarzinom nach der Thyreoidektomie ist. 78.724 Patienten, die in den Jahren 1998 bis 2012 in der US-amerikanischen Krebsdatenbank registriert waren, wurden in die Studie aufgenommen. 38.653 (49,1\%) von ihnen hatten befallene Lymphknoten und wurden für die Risikoabschätzung falsch-negativer Ergebnisse herangezogen.

Anhand dieser Daten errechneten die Forscher die Wahrscheinlichkeit, mit der Patienten trotz befallener Lymphknoten fälschlicherweise als nodalnegativ angesehen wurden: Sie lag bei $53 \%$, wenn nur ein einziger Lymphknoten untersucht wurde, und sank auf unter $10 \%$, wenn mehr als sechs Lymphknoten untersucht wurden.

Anschließend wurde die tatsächliche Prävalenz positiver Lymphknoten in der gesamten Studienpopulation berechnet. Sie variierte zwischen $36,9-78,6 \%$ in den Stadien T1b-4 und war unter Berücksichtigung falsch-negativer Befunde deutlich höher (47,1-98,4\%).

Zuletzt berechneten die Forscher, wie viele Lymphknoten untersucht werden müssen, um einen übersehenen Lymphknotenbefall mit 90\% Konfidenz auszuschließen: Bei Patienten mit einer T1bErkrankung waren es sechs Lymphknoten, bei Patienten mit einer T2-Erkrankung neun und bei Patienten mit einer T3-Erkrankung 18 Lymphknoten. Sensitivitätsanalysen ergaben, dass drei, vier bzw. acht Lymphknoten untersucht werden müssen, um eine vergleichbare Verlässlichkeit der Lymphknotenbeurteilung zu erzielen.

Judith Neumaier

Robinson TJ et al. How Many Lymph Nodes Are Enough? Assessing the Adequacy of Lymph Node Yield for Papillary Thyroid Cancer. J Clin Oncol. 2016;34(28):3434-9. 